\title{
A Design and Implementation of Simple Spectrum Analyzer Based on DDS
}

\author{
Hang $\mathrm{Yu}^{1}$, Sun Kexue ${ }^{1,2, a}$, Liu Yanming ${ }^{1}$, Zhu Siqing ${ }^{1}$ and Cheng Xiefeng ${ }^{1,2, b}$ \\ ${ }^{1}$ School of Electronic Science and Engineering, Nanjing University of Posts and \\ Telecommunications, Nanjing, 210023, China \\ ${ }^{2}$ Jiangsu Province Engineering Lab of RF integration \& Micropackage, Nanjing, 210023, China \\ a email: sunkx@njupt.edu.cn, ${ }^{\mathrm{b}}$ email: chengxf@njupt.edu.cn
}

Keywords: Spectrum analyzer; DDS; STM32; Swept-frequency.

\begin{abstract}
In this paper, we design a basic spectrum analyzer which can analyze the signal with the bandwidth in $10 \mathrm{MHz} 100 \mathrm{MHz}$ and the minimum resolution in $100 \mathrm{KHz}$. We utilize the AD9854 DDS (Direct Digital Synthesizer), STM32F407, AD831, AD835 and the TFT LCD to design and implement a system that has menu-driven function selection mode and better man-machine interface. This system and actual test results showed that this spectrum analyzer can achieve the design goal. It reduces the cost and is portable with low power consumption.
\end{abstract}

\section{Introduction}

Nowadays a spectrum analyzer is widely used in a lot of fields but dedicated equipment is quite expensive. We need to find a low-cost and general design to achieve spectrum analyzer which engineers and scientists can implement by themselves easily.

Some designs of spectrum analyzer are based on high speed ADC and FPGA to get spectrum by FFT. But the bandwidth limited by the speed of ADC is not very wide, so it is expensive without generality. Some designs use PLL based on VCO, but it is hard for individual to achieve and expensive. We need to find a way to solve these problems [1, 2].

So we chose DDS to realize this system. The direct digital frequency synthesizer (DDS) has characteristics of strong resolving power, quickly switches and abroad applications. This technology can help us to design with low cost and easy implementation for individual.DDS AD9854 can generate sine signal from 0 to $120 \mathrm{MHz}$. However, when the frequency reaching 60 $\mathrm{MHz}$, the amplitude of the signal will decline sharply. Therefore, we just use the frequency from 0.5 $\mathrm{MHz}$ to $50 \mathrm{MHz}$. Then, we can utilize an AD835 as multiplier which can double frequency. Local oscillator made of DDS combined with multiplier will be controlled by STM32 to sweep from 1 to $100 \mathrm{MHz}$, with the step of $100 \mathrm{KHz}$ [3-5].

In this paper DDS which is controlled by STM32F407 will sweep frequency to detect other signals. It's inexpensive and easy to realize for scientists and engineers.

\section{Overall design}

We use a touch screen as a console to operate the whole system. STM32 will execute the command from screen to control DDS to sweep.

The swept-frequency signal will be mixed with detection signal by AD831.The mixed signal will pass a $100 \mathrm{KHz}$ low-pass filter to get the low frequency component. Then we use the ADC of STM32 to detect the low frequency signal.

STM32 can analyze the frequency of detection signals according to the feedback signal measured by the ADC of STM32. 


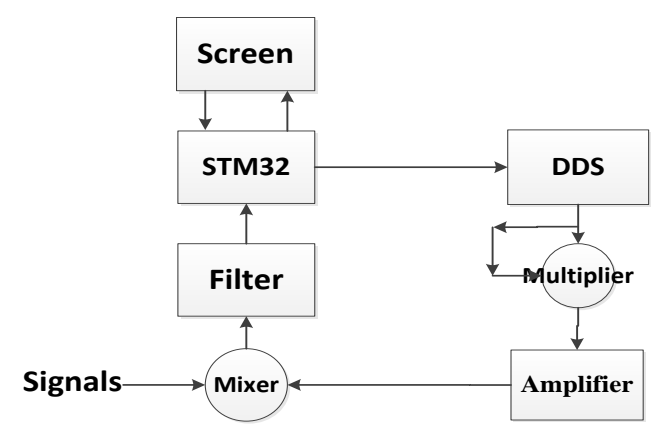

Fig.1. The system's general design

\section{Theoretical analysis and calculation}

The theory of ADC

To measured accurately, we decide give ADC 1ms to detect low-frequency signal. Now we need to calculate the lowest frequency that the ADC could measured accurately.

We use $36 \mathrm{MHz}$ ADC clock and 12bits sampling. It's sampling frequency is

$\mathrm{f}_{\text {sam }}=\frac{\mathrm{f}_{\text {adc }}}{15}=2.4 \mathrm{Mhz}$

So the ADC will sample 2400 times in a $1 \mathrm{~ms}$.

To get more accurate data, we need to sample at least 10 cycles.

$\mathrm{f}_{\text {min }}=\frac{10}{\mathrm{~T}}=10 \mathrm{khz}$

So the lowest frequency we could measure is $10 \mathrm{khz}$. We had to abandon the formal plan which uses a $100 \mathrm{KHz}$ low-pass filter. We replace it with a $10 \mathrm{KHz} 110 \mathrm{KHz}$ band-pass filter.

The design of amplifier

The amplitude of signal generated by AD9854 is about $500 \mathrm{mV}$, the output signal of multiplier will decline to $125 \mathrm{mV}$, so we need to design a high frequency amplifier. It's about 4 times gain.

Multiplier

The multiplier depends on AD835.

If input signal $\mathrm{X}$ and $\mathrm{Y}$ are sine wave [3],

$$
W=X Y=(A \sin \omega t)(A \sin \omega t)=\frac{A^{2}}{2}(1-\cos 2 \omega t)
$$

So at the rear of multiplier, we should attach a capacitor to filter DC component and an amplifier to Amplify signal. And we'll get double frequency signal that we need.

The mixer depends on AD831. Functional block diagram of AD831 is shown in Fig. 2.

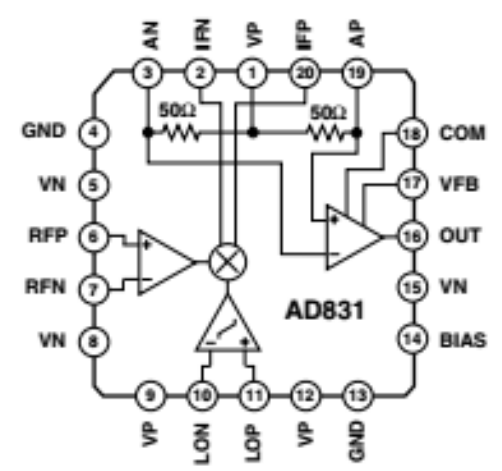

Fig. 2.Functional block diagram of AD831

When the integral output amplifier is used, pins IFN and IFP are connected directly to pins AFN and AFP; the on-chip load resistors convert the output current into a voltage that drives the output amplifier. The ratio of these load resistors to resistors R1, R2 provides nominal unity gain (0 dB) 
from RF-to-IF. The expression for the gain, in decibels, is

$$
\mathrm{G}_{\mathrm{dB}}=20 \lg \left(\frac{\pi}{2}\right)\left(\frac{1}{2}\right)\left(\frac{4}{\pi}\right)
$$

Where: $4 / \pi$ is the amplitude of the fundamental component of a square wave. $1 / 2$ is the conversion loss, and $\pi / 2$ is the small signal dc gain of the AD831 when the LO input is driven fully positive or negative.

The swept signal source based on DDS technique consists of a reference frequency source, a phase accumulator, and a RAM used to store the sine sampling points.

We set the frequency of the reference frequency source as $f_{c l k}$ and the counter capacity of the phase accumulator as $2^{N}$ ( $\mathrm{N}$ is the digit of the accumulator). If the frequency control word is $\mathrm{M}$, then the frequency of the DDS system's output signal is[5][6]

$$
f_{\text {out }}=\frac{f_{c l k}}{2^{N}} \times M
$$

The $f_{c l k}$ that we use is $300 \mathrm{Mhz}$. Then we can calculate the frequency control word according to equality(5).

The detection cycle of the software is shown in Fig. 3.

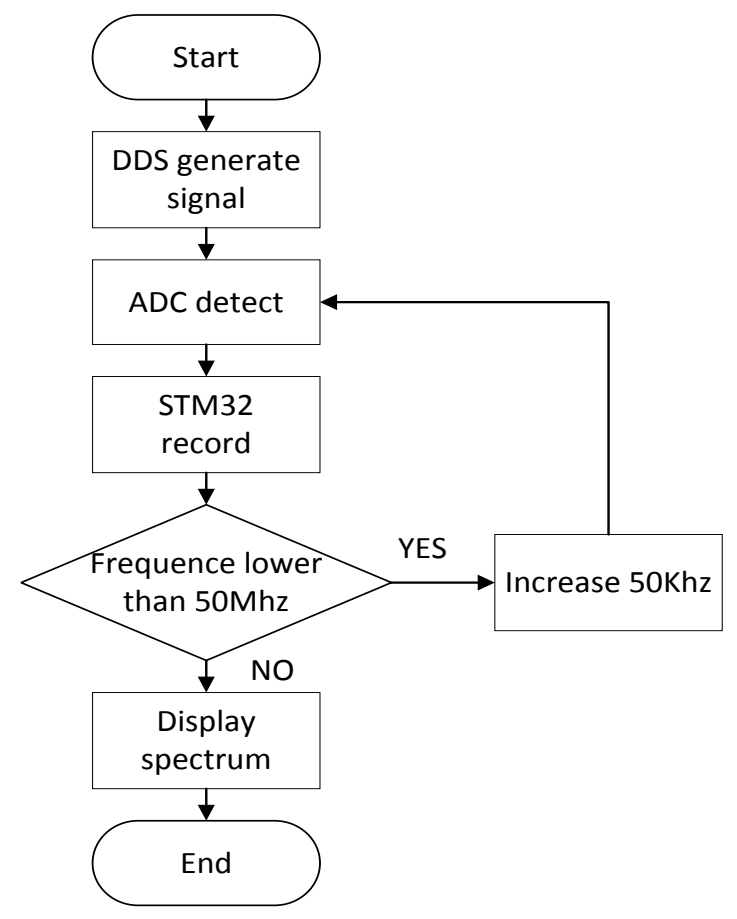

Fig. 3. Detection cycle of software

\section{Circuit design}

The design of $10 \mathrm{k} \sim 110 \mathrm{khz}$ band-pass filter

This filter need to have a sharp edge,but it don't need to care about group delay,so we choose Chebyshev filter.

We use the ADC of STM32 with 12 bits Precision.Under the 3.3V reference voltage,the minimum voltage could be detected is

$$
V_{\min }=\frac{V_{\mathrm{Re} f}}{4096}=0.8 \mathrm{mV}
$$

Vmax is about $500 \mathrm{mV}$,so stopband Attenuation is

$$
A_{s b}=20 \lg \frac{V_{\min }}{V_{\max }}=-56 \mathrm{~dB}
$$


With the help of aided design software, we get a filter as shown in Fig.4, and according to ADI's reference design, we design the circuit as shown in Fig.5,

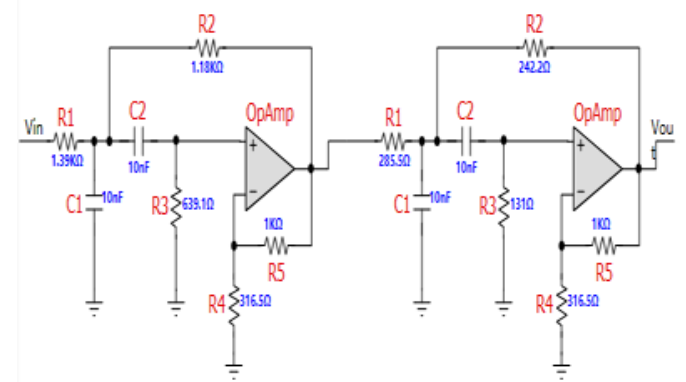

Fig. 4. Circuits of band-pass filter

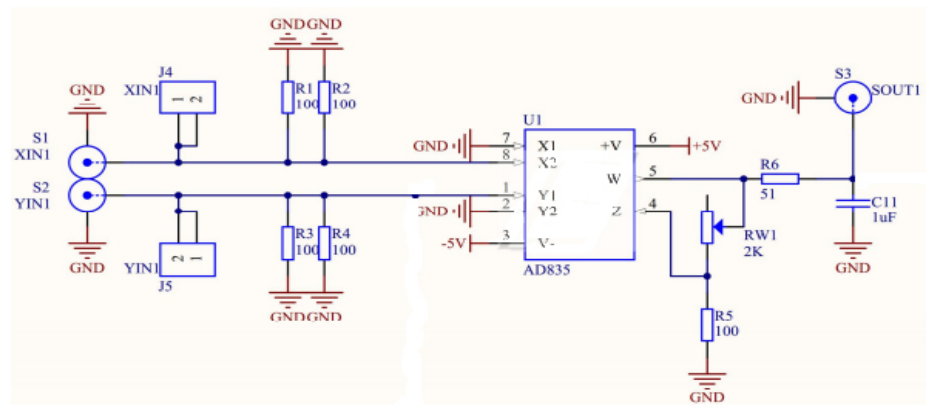

Fig. 5. Circuit of multiplier

\section{Results}

The test of point frequency is listed in Table 1. We can see that the system is hard to identify the signal like $1.99 \mathrm{MHz}$ from the Table 1.To improve the precision of this system, we must design a better filter with a sharper edge. Because of the minimum resolution is $100 \mathrm{kHz}$, so the test of point frequency is not very accurate.

The Table 2 lists the AM wave test data. From this table we can see that, this spectrum Analyzer can identify different frequency of a complex signal.

Table 1.Point frequency test

\begin{tabular}{|cccc|}
\hline \multicolumn{4}{|c|}{ Point frequency test } \\
\hline Fre $/ \mathrm{MHz}$ & Measure1/MHz & Measure2/MHz & Measure3/MHz \\
\hline 1.10 & 1.1 & 1.1 & 1.1 \\
\hline 1.11 & 1.1 & 1.1 & 1.1 \\
\hline 1.19 & 1.1 & 1.2 & 1.2 \\
\hline 1.20 & 1.2 & 1.2 & 1.2 \\
\hline 50.00 & 50.0 & 50.0 & 50.0 \\
\hline 90.00 & 90.0 & 90.0 & 90.0 \\
\hline 99.90 & 99.9 & 99.9 & 99.9 \\
\hline 99.91 & 99.9 & 99.9 & 99.9 \\
\hline 99.99 & 100.0 & 99.9 & 100.0 \\
\hline 100.00 & 100.0 & 100.0 & 100.0 \\
\hline
\end{tabular}

Table 2.AM wave test

\begin{tabular}{|cccc|}
\hline \multicolumn{4}{c|}{ AM wave test } \\
\hline $\begin{array}{c}\mathrm{FC} \\
(\mathrm{MHz})\end{array}$ & $\begin{array}{c}\mathrm{FW} \\
(\mathrm{MHz})\end{array}$ & $\begin{array}{c}\text { Lower sideband } \\
(\mathrm{MHz})\end{array}$ & $\begin{array}{c}\text { Upper sideband } \\
(\mathrm{MHz})\end{array}$ \\
\hline 50.0 & 0.1 & 49.9 & 50.1 \\
\hline 90.0 & 0.2 & 89.8 & 90.2 \\
\hline 99.0 & 1.0 & 98.0 & 100.0 \\
\hline
\end{tabular}


To analyze the system's ability of identify different frequency component, we use AM signal to test it. The spectrum chart of AM wave is shown in Fig. 6. The test result as shown in Fig. 7.

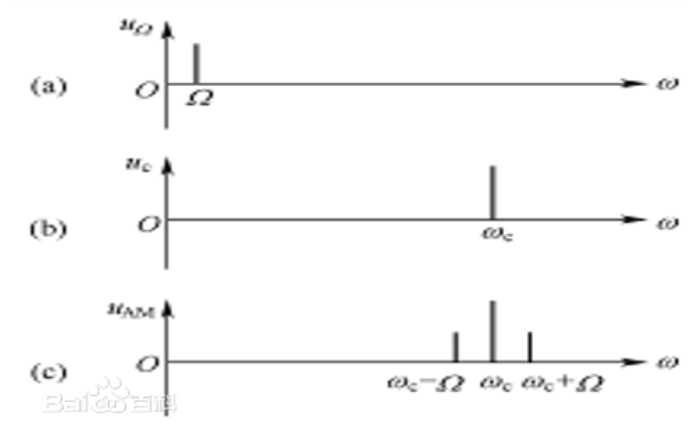

Fig. 6.AM wave’s spectrum chart

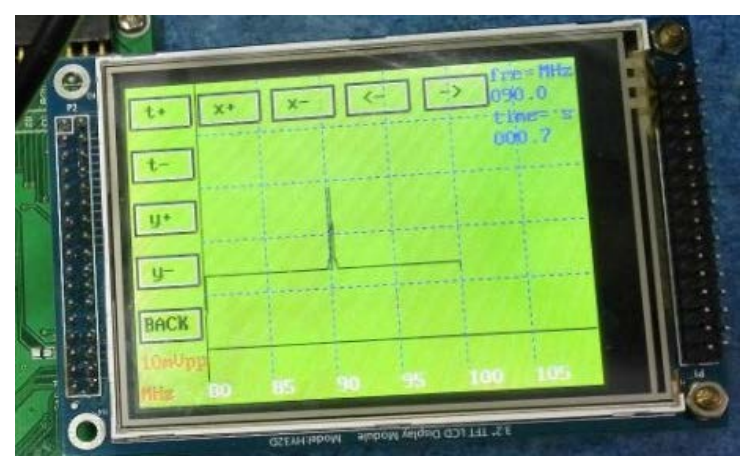

Fig. 7. spectrum chart in practical test

\section{Conclusion}

The paper focuses on a design that realizes the DDS technology through the use of STM32. It achieves the goal that designs a spectrum analyzer which is inexpensive and convenient. This design can be applied on the a few of fields such as communication systems and scientific researches.

\section{Acknowledgement}

This research was financially supported by the National Natural Science Foundation of China (Grant No.61271334), Natural Science Foundation of the Jiangsu Higher Education Institutions of China (Grant No. 15KJD510001), and Natural Science Foundation of Nanjing University of Posts and Telecommunications (Grant No. NY214049).

\section{Reference}

[1] Mingyu Gao. Yunfei Liu. Jiye Huang. Zhiwei He, Design Of The Automatic Jacquard Control System Based On STM32F407, International Conference on Information Science, Electronics \& Electrical Engineering, 2014(2), 1143-1146.

[2] Wen-hui Chen. Fei Jia. Design of spectrum analyzer based on DDS, International Electronic Elements, 2008(12), 24-25.

[3] Chong Yao. Yueqiang Lu. Weilin Zhang, Communication Experiment Dedicated Digital Spectrum Analyzer,10.1109/WNIS.2009.26,183 - 185.

[4] Zeinert Martin and Makula Petr, Impact Assessment of Power Electronics on Experimental Aircraft Receiver, 2015,10.1109/MILTECHS.2015.7153740,1-5.

[5] Hongzhen YU. Communication electronic circuit. Tsinghau University Press, 2012(9). 